\title{
Variant Under Investigation (VUI) and Severity of COVID-19 Third Wave. Is there a Correlation?
}

\author{
Sara $\mathrm{AS}^{1,2}$, Mohamed Raslan ${ }^{1,2}$, Eslam MS ${ }^{2}$ and Nagwa A Sabri ${ }^{1 *}$ \\ ${ }^{1}$ Department of Clinical Pharmacy, Faculty of Pharmacy, Ain Shams University, \\ Cairo, Egypt \\ ${ }^{2}$ Drug Research Centre, Cairo, Egypt \\ *Corresponding Author: Nagwa A Sabri, Department of Clinical Pharmacy, Faculty \\ of Pharmacy, Ain Shams University, Cairo, Egypt.
}

Received: August 24, 2021

Published: October 23, 2021

(C) All rights are reserved by Nagwa A Sabri., et al.

\begin{abstract}
Coronavirus (COVID-19) was introduced into society by the end of 2019, and there have already been over 160 million infections and 3.5 million fatalities as a result of it. Sustainable and non-declinable waves of covid-19 may result in different viral mutations and the emergence of different variants. The second wave as well as the third wave showed to be highly spreadable and in some countries mortality rates were higher in the third wave. Different variants emerged and were contributory in the second wave of covid-19 like 501Y.V2 in South Africa. Besides, in Spain the B.1.1.7 strain which is highly transmissible was contributory for the third wave. Moreover, by the end of May 2021 a new variant VUI-21MAY-02 (C.36.3) was discovered in United Kingdom which has an Egyptian origin and no proven evidence that it contributes to high incidences or increase case severities. Furthermore, mortality rates in Egypt showed to be significantly higher than the worldwide average mortality rates, indicating the need for more attention regarding protective measures, social distancing, and other precautionary procedures.
\end{abstract}

Keywords: Coronavirus Disease 2019; Severe Acute Respiratory Syndrome Coronavirus 2; Third Wave; Immunization; C.36.3 Variant

\section{Abbreviations}

WHO: World Health Organization; COVID-19: Coronavirus Disease 2019; SARS-CoV-2: Severe Acute Respiratory Syndrome Coronavirus 2; US (CDC): United States Center of Disease Control; VUI: Variant Under Investigation; SGTF: S-gene Target Failure.

\section{Introduction}

The World Health Organization's (WHO) China Country Office was contacted in late December 2019 of cases of pneumonia of unknown aetiology in Wuhan, China. The source of this pneumonia was eventually revealed to be a new virus called as severe acute respiratory syndrome coronavirus 2 (SARS-CoV-2) that causes coro- navirus disease 2019 (COVID-19). The WHO declared COVID-19 as a pandemic on March 11, 2020 [1].

Data from WHO revealed that the second wave of covid-19 in Egypt started at the beginning of October 2020, and peaked at the end of December 2020 and January 2021. Unfortunately, this wave didn't decline, and mortality rate is still high.

The second wave of infections and the emergence of new, more transmissible strains of the virus [2], together with public noncompliance with social distancing and protective measures were all a contributing factors for sustaining the second wave and non- 
declining mortalities. We can say that a third wave of COVID-19 in Egypt overlapped the second wave by February 2021 and peaked by the mid of May 2021.

The initial wave in Egypt, like the rest of the globe, dropped dramatically around the beginning of October, making Egyptians more complacent and unwilling to take even the most basic measures. The individual's reckless ease of the constrains, relaxing attitude, and carelessness in taking the required protective measures; believing that the seriousness of the condition had passed as it was the beginning to notice that many citizens were not adhering to the safety measures implied by the authorities, on the other hand, some healthcare providers feel that the government decided on an early reopening in order to avert a financial catastrophe among its residents, the impact of that policy shift will take a month or more to be observed. Furthermore, numerous cycles of infection/recovery must occur before a discernible rise appears in the data [3].

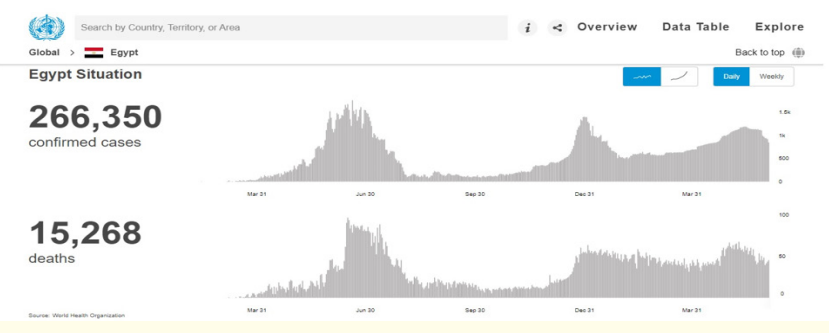

Figure 1: WHO representative charts for covid-19 Egypt situation by 6-6-2021 [4].

The consequences of sustaining and overlapping different covid-19 waves and increasing number of infections definitely can contribute to the emergence of new covid-19 variants that may be possess unpredictable spreading pattern, disease severity, and unknown impact on current immunization effectiveness.

\section{Discussion}

COVID-19's first clinical signs are comparable to those of other kinds of viral pneumonia, with varied degrees of severity. The incubation time is typically between 3 and 7 days [US (CDC) estimates a range of 2 to 14 days], with the shortest being 1 day and the great majority occurring within 2 weeks. Subjects may continue to be as- ymptomatic. The earliest usual symptoms of COVID-19 pneumonia were fever, cough, and shortness of breath, while chills, muscular discomfort, sore throat, and new loss of taste and smell were later added to the list. Some people suffer headaches and myalgia, while others may have diarrhea, indicating that the gastrointestinal tract is involved. Patients with severe symptoms typically have chest tightness and dyspnea approximately 7 to 10 days after beginning of symptoms, with a proportion progressing to acute respiratory distress syndrome, septic shock, metabolic acidosis, and coagulopathy [5].

On the basis of the mutation profile and increasing importation from a wider international region, the variant VUI-21MAY-02 (C.36.3) was identified as a Variant Under Investigation (VUI) on May $24^{\text {th }} 2021$ in the United Kingdom. Too far, 109 cases of VUI21MAY-02 (C.36.3) variant have been reported across the UK country. The C.36.3 variant was discovered for the first time in Thailand, in individuals that had travelled from Egypt. Up to date of presenting this review, there is no indication that this variant causes more severe illness or makes existing immunizations less effective [6].

The VUI-21MAY-02 (C.36.3) variant has numerous spike receptor binding domain (RBD) mutations associated with antigenic alteration, including L452R and R346S. It also has N terminal domain (NTD) mutations that may be related with antigenic alteration, such as S12F and W152R. It also has deletions 69-70 and Q677H. Thermofisher TaqPath test targets three SARS-CoV-2 regions: ORF1ab, N, and S-genes. Deletion 69-70 is related with S gene target failure (SGTF) in the TaqPath test, which is utilized in surveillance analysis indicating that SGTF is a marker for the new SARS-CoV-2 variant. Different sequences from 34 countries were identified by 26th May 2021. For instance, there was 148 sequences from Germany, 144 from USA, 62 from Switzerland, and 33 from Egypt $[7,8]$.

In South Africa, the second wave was associated with a greater incidence and faster increase in hospitalizations, as well as an increase in in-hospital mortality. While some of this might be attributed to increased demand on the health-care system, an additional rise in hospitalized patient mortality might be attributed to the new strain 501Y.V2. Peak rates of COVID-19 cases, admissions, and in-hospital mortality (per 100,000 people) in the second wave surpassed those in the first wave which was 138.1 against $240.1 ; 16.7$ against 28.9; and 3.3 compared to 7.1, respectively [9]. 
The epidemiologic characteristics of the second and third waves of COVID-19 pandemic in South Korea revealed that the third wave was characterized by delayed strengthening of social distancing policies ( 3 versus 15 days), longer duration (36 versus $>56$ days), and a higher case fatality rate ( 0.91 percent versus 1.26 percent). Transmission chains differed significantly between the second and third waves. When compared to the second wave, the proportion of local clusters was lower in the third wave (24.8 percent versus 45.7 percent), whereas personal contact transmission (38.5 percent versus 25.9 percent) and unknown transmission pathways (23.5 percent versus 20.8 percent) were greater [10].

The situation may be different in Spain. After Christmas 2021, Madrid City had a third wave of COVID-19. Six main differences between this wave and prior waves were noted. First, household contacts accounted for a sizable number of cases. Second, having availability to quick antigen testing enabled for faster diagnosis and isolation. Third, the number of clinically severe cases and the fatality rate were decreased. Fourth, the more transmissible B.1.1.7 strain was becoming more common. Fifth, the advantages of immunization have been observed in healthcare professionals and nursing homes. And finally, reinfections became increasingly prevalent. By 17 April 2021, roughly 25\% of Madrid's population had been infected with covid-19 [11].

The situation in Egypt is considered similar in the third wave as the second wave regarding disease symptoms severity, incidence and death rates. The mortality rate showed to be of $5.1 \%$ versus $4 \%$ (third wave versus second wave respectively). The cumulative death rate is Egypt is about 5.75\% which is higher than the worldwide cumulative death rate (2.08\%), USA (1.78\%), UK (2.8\%), and Saudi Arabia (1.63\%) [12]. On the other hand, the number of vaccinated individuals in Egypt with at least one dose is about 2.4\% [13].

\section{Conclusion}

We can assume that the second and third waves worldwide are sharing the same characteristics as the third waves overlapped the second wave, therefore, safety and protective measures should be adhered to by the whole population. Besides, mass vaccination is required to attain, as much as possible, lower mortality rates, hospital admissions, and decrease the severe symptoms. Till the moment no indication that the VUI-21MAY-02 (C.36.3) variant showed more severe symptoms or affect immunization efficacy. As all the investigation was done in the United Kingdom, thus, it is recommended that an extensive investigations and studies are required to discover the significance of (C.36.3) on the incidence, mortality rates, prevalence, and SARS-COV-2 resistance to the different management protocols used leading to an increase in symptoms severity an mortality rates in the future.

\section{Funding Sources}

This research did not receive any specific grant from funding agencies in the public, commercial, or not-for-profit sectors.

\section{Declarations of Interest}

None.

\section{Acknowledgements}

The authors would like to present their deep gratitude and thankfulness to Drug Research Centre - Cairo/Egypt for giving plenty of time and support to the researchers to share and participate in this work.

\section{Bibliography}

1. Rothan HA and Byrareddy SN. "The epidemiology and pathogenesis of coronavirus disease (COVID-19) outbreak". Journal of Autoimmune 109 (2020): 102433.

2. Villoutreix BO., et al. "In silico investigation of the new UK (B.1.1.7) and South African (501y.v2) SARS-CoV-2 variants with a focus at the ace2-spike rbd interface". International Journal of Molecular Sciences 22 (2021): 1-13.

3. Deif AS and El-Naggar SA. "Modeling the COVID-19 spread, a case study of Egypt". Journal of the Egyptian Mathematical Society 29.1 (2021): 13.

4. https://covid19.who.int/region/emro/country/eg

5. Wang C., et al. "COVID-19 in early 2021: current status and looking forward". Signal Transduction and Targeted Therapy 6 (2021): 114.

6. https://www.gov.uk/government/news/confirmed-cases-ofcovid-19-variants-identified-in-uk 
7. Public Health England (PHE). SARS-CoV-2 variants of concern and variants under investigation in England, Technical briefing 13, 27 May 2021, This briefing provides an update on previous briefings up to 22 May (2021).

8. Alfredo Maria Gravagnuolo., et al. "Epidemiological Investigation of New SARS-CoV-2 Variant of Concern 202012/01 in England". medRxiv (2021).

9. Waasila Jassat., et al. "Increased mortality among individuals hospitalised with COVID-19 during the second wave in South Africa”. medRxiv (2021).

10. Hye Seong., et al. "Comparison of the second and third waves of the COVID-19 pandemic in South Korea: Importance of early public health intervention". International Journal of Infectious Diseases 104 (2021): 742-745.

11. Vicente Soriano., et al. "Third wave of COVID-19 in Madrid, Spain". International Journal of Infectious Diseases 107 (2021): 212-214.

12. Weekly epidemiological update on COVID-19 - 1 June (2021).

13. https://covid19.who.int/region/emro/country/eg

Volume 5 Issue 11 November 2021

(C) All rights are reserved by Nagwa A Sabri., et al. 